\title{
Transition of Mental Health Service Delivery to Telepsychiatry in Response to COVID-19: A Literature Review
}

\author{
Hua $\mathrm{Li}^{1}{ }^{\mathbb{D}} \cdot$ Alana Glecia $^{2} \cdot$ Arlene Kent-Wilkinson $^{1} \cdot$ Donald Leidl $^{1} \cdot$ Manal Kleib $^{3}$. \\ Tracie Risling ${ }^{1}$
}

Accepted: 30 May 2021 / Published online: 8 June 2021

(c) The Author(s), under exclusive licence to Springer Science+Business Media, LLC, part of Springer Nature 2021

\begin{abstract}
Due to COVID-19, face-to-face mental health service delivery has been interrupted by social distancing and stay-at-home orders. To abridge physical distance between patients and healthcare providers, while limiting exposure to COVID-19, telepsychiatry has been widely adopted to provide services to patients with pre-existing mental health disorders. Though telepsychiatry has become more mainstream in delivering mental health services during COVID-19, evaluation studies of the rapid conversion of care delivery from face-toface to telepsychiatry have been limited. The aim of this study was to review the literature on the transition of mental health service delivery to telepsychiatry during COVID-19. The findings of the current review showed that a majority of patients and healthcare providers were satisfied with telepsychiatry services, and suggest that telepsychiatry is feasible and appropriate for supporting patients and healthcare providers during COVID-19.
\end{abstract}

Keywords COVID-19 $\cdot$ Mental health service delivery $\cdot$ Telepsychiatry $\cdot$ Patients · Healthcare providers

Hua Li

hua.li@usask.ca

Alana Glecia

agd262@mail.usask.ca

Arlene Kent-Wilkinson

arlene.kent@usask.ca

Donald Leidl

donald.leidl@usask.ca

Manal Kleib

manal.kleib@ualberta.ca

Tracie Risling

tracie.risling@usask.ca

1 College of Nursing, University of Saskatchewan, Health Sciences Building, E-Wing, 104 Clinic Place, Saskatoon, SK S7N 2Z4, Canada

2 Department of Sociology, University of Saskatchewan, Arts Building, 9 Campus Drive, Saskatoon, SK S7N 5A5, Canada

3 Faculty of Nursing, University of Alberta 5-112 Edmonton Clinic Health Academy, Edmonton, AB T6G 1C9, Canada 


\section{Introduction}

The World Health Organization (WHO) declared COVID-19 a global pandemic on March 11, 2020 [1]. In an effort to control the spread of the virus, many countries have adopted various measures including social distancing, stay-at-home orders, closure of nonessential business, travel restrictions, and quarantine [1]. Although these measures have been useful for infection control, they also created new challenges and population strain. This has been especially evident for those with pre-existing mental health conditions with the impact of this further compounded by difficulty accessing healthcare services [2]. As mental healthcare is vital to symptom management for many patients, accessing healthcare services is even more critical in the midst of the pandemic.

To provide mental healthcare to those who need it most, while simultaneously limiting exposure to COVID-19, many healthcare providers turned to telehealth as an effective way to abridge physical distance between patients and healthcare providers. Telehealth is defined as the use of technology via audio and video telecommunications in order to provide healthcare across geographical distances, to facilitate the exchange of information between healthcare providers, or provide healthcare when it is not possible to have face-to-face contact $[3,4]$. When applied to mental health, these methods are known as telepsychiatry, which includes teleconsultation, teletherapy, telepsychology, telepsychotherapy, or telemental health via videoconferencing, phone conversations, and real-time chat [5].

Telepsychiatry is not a new concept, rather an underused technology before the pandemic. Based on a population study in the U.S., telemedicine visits were 0.02 per 1000 in 2005 and increased to 6.57 per 1000 in 2017 , including a $38 \%$ growth in primary health care and $56 \%$ growth in telemental health with the highest rate increases in geographic areas where there were no psychiatrists [6]. In terms of virtual health worldwide, prior to COVID-19, various organizations and governments had recognized the potential of virtual health's capacity to shape healthcare delivery, especially improving accessibility [7-9]. In the wake of COVID-19, social distancing and stay-at-home order as methods to prevent the spread of the virus have forced many patients and healthcare providers to rapidly adopt telepsychiatry as a response to these unprecedented challenges. Though telepsychiatry has become more mainstream in delivering mental health services during COVID-19 [10,11], evaluation studies of the rapid conversion of care delivery from face-to-face to telepsychiatry have been limited.

The aim of this study was to review the literature on the transition of mental health service delivery from face-to-face to telepsychiatry in response to COVID-19, especially from the perspectives of both patients and healthcare providers. The review answered the following two questions: (1) Did the transition of mental health service delivery to telepsychiatry meet the expectation of service users (patients) and services providers during COVID-19? (2) Was telepsychiatry a reliable and feasible method to provide mental health services during COVID-19? 


\section{Methods}

\section{Search Strategy and Inclusion and Exclusion Criteria}

A literature search for contemporary literature with a focus on the transition of mental health service delivery from face-to-face to telepsychiatry during the COVID-19 pandemic was conducted in three databases: PubMed, PsycInfo, and Web of Science. Initial search terms developed were associated with patients with mental health disorders, tele* (e.g., telehealth, telemedicine, telepsychiatry, telepsychology, telenursing), and COVID-19. As we were interested in patients who had existing mental health disorders prior to (i.e., not caused by) the COVID-19 pandemic, an exhaustive list of exclusionary terms was included in the search using the 'NOT' Boolean operator.

Inclusion criteria for the search included all types of research articles (e.g., quantitative, qualitative, or mixed methods) that focused on (1) transition of mental health services to telepsychiatry due to COVID-19; (2) patients with pre-exsiting mental health disorders $\geq 18$ years old, and/or mental healthcare providers; (3) were published between January 2020 and January 2021; and (4) were peer-reviewed English-language publications. Exclusion criteria included (1) studies that did not examine the experiences or outcomes of the transaction from the perspectives of patients and/or healthcare providers; (2) study population < 18 years old; (3) published in non-English language; (4) non-peer reviewed articles (i.e., gray literature); and (5) published outside specified publication year limit.

\section{Screening and Selection}

Titles and abstracts were screened to assess the relevance. If a seemingly relevant title did not have an abstract, the full text of the article was reviewed. Articles that met the inclusion criteria, were moved on to the next step of full text review, during which authors also handsearched the bibliography of all relevant sources. Articles that survived the full text review, proceeded to data extraction.

\section{Data Extraction}

Relevant information of each selected article was extracted and entered into a standard form: Author (year), country; type of study design, aim of study, sample population and size, measures of assessment, interventions, and major findings.

\section{Results}

\section{Study Selection}

A total of 806 articles were retrieved. After removing duplicates, a total of 590 studies underwent title and abstract screening. Of these 590, 21 articles were relevant and proceeded for full text review. Upon full text review, a total of eight studies met the inclusion criteria, and were selected for data extraction. All selected articles had a focus on the transition of mental health service delivery to telepsychiatry during the COVID-19 pandemic. 


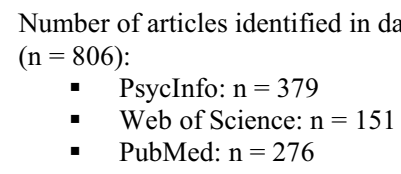

After duplicates removed, $\mathrm{n}=590$

Title and abstract screening: $\mathrm{n}=590$

Studies identified as relevant to the topic, with full text review: $n=21$

Studies included in the review: $n=8$

Fig. 1 Study selection flow diagram

Hand searching of reference lists of included studies did not retrieve additional results. A study selection flow diagram is presented in Fig. 1.

Studies excluded $(\mathrm{n}=13)$ based on not meeting criteria in the full-text review:

- Not related to telehealth delivery of mental health services during COVID-19 $(\mathrm{n}=7)$

- Not a research study (e.g., commentary; protocol) $(\mathrm{n}=6)$

\section{Summary of Study Characteristics}

All studies were published in 2020. A majority of studies were conducted in the U.S. [10-15], while Spain and the Dominican Republic were the countries of one study each, respectively $[16,17]$. Six studies were quantitative, while one employed qualitative methods [15], and another study used mixed methods [14]. Of eight selected studies, six focused exclusively on patient experiences or patient information of the transition of mental health service delivery to telepsychiatry $[10-12,14,16,17]$, while two studies examined healthcare providers' perspectives of the transition $[13,15]$.

A total of 7,655 participants were involved in the selected studies including 4,316 patients, 3,307 healthcare providers, and 32 patient proxies/parents (56.3\% patients; $43.2 \%$ service providers; $0.5 \%$ patient proxy/parents respectively), which provide a more comprehensive perspective of experiences with the transition of mental health service delivery to telepsychiatry during the COVID-19 pandemic. A summary of selected studies is presented in Table 1. 


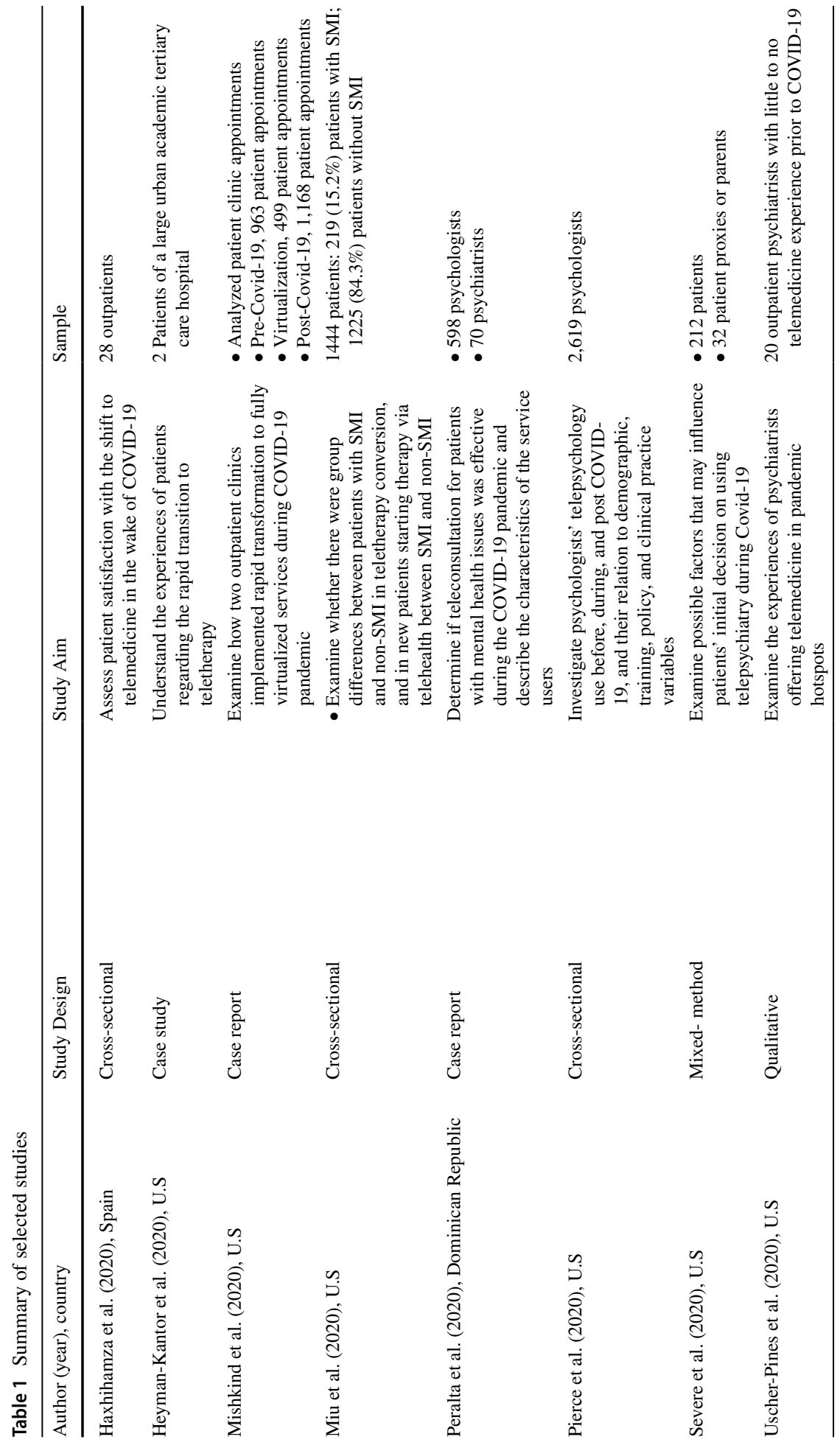




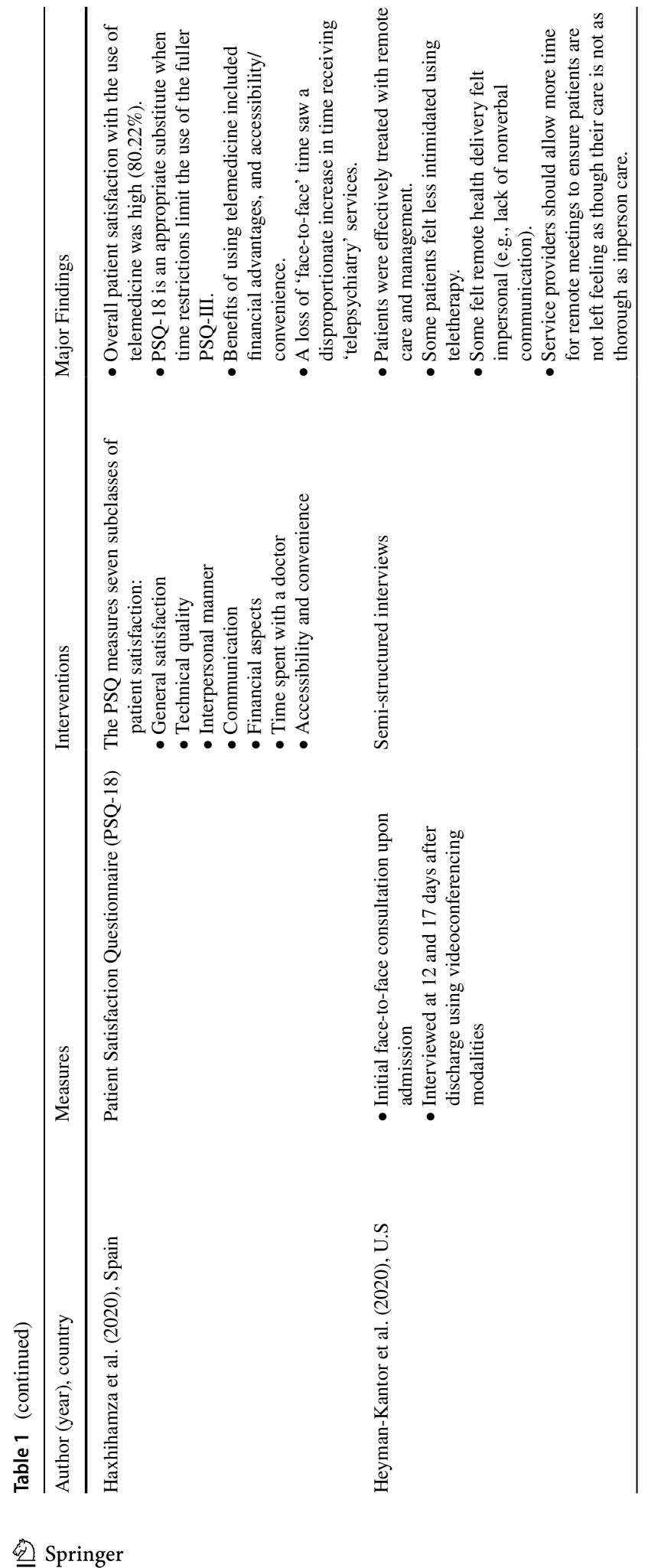




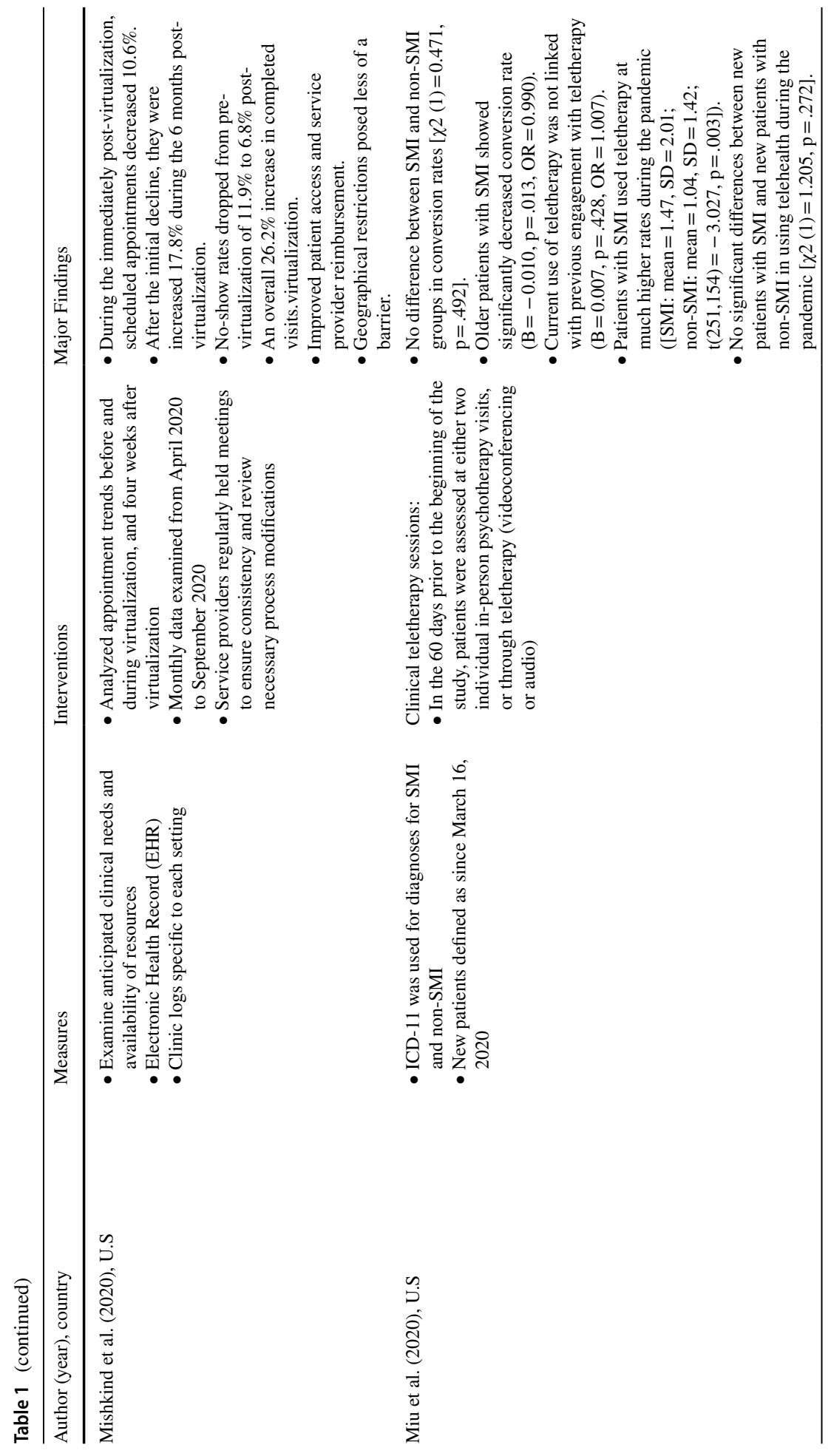




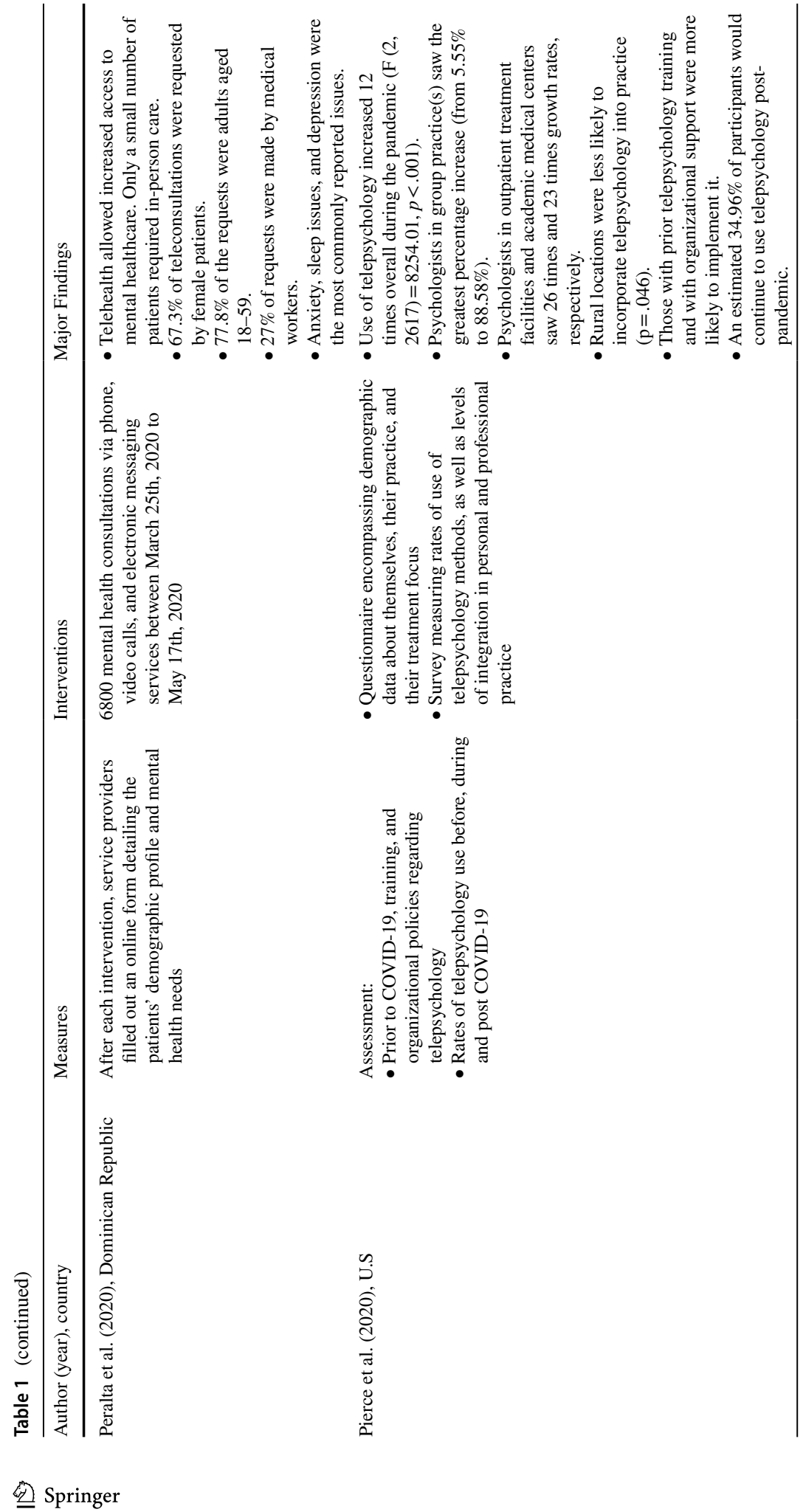




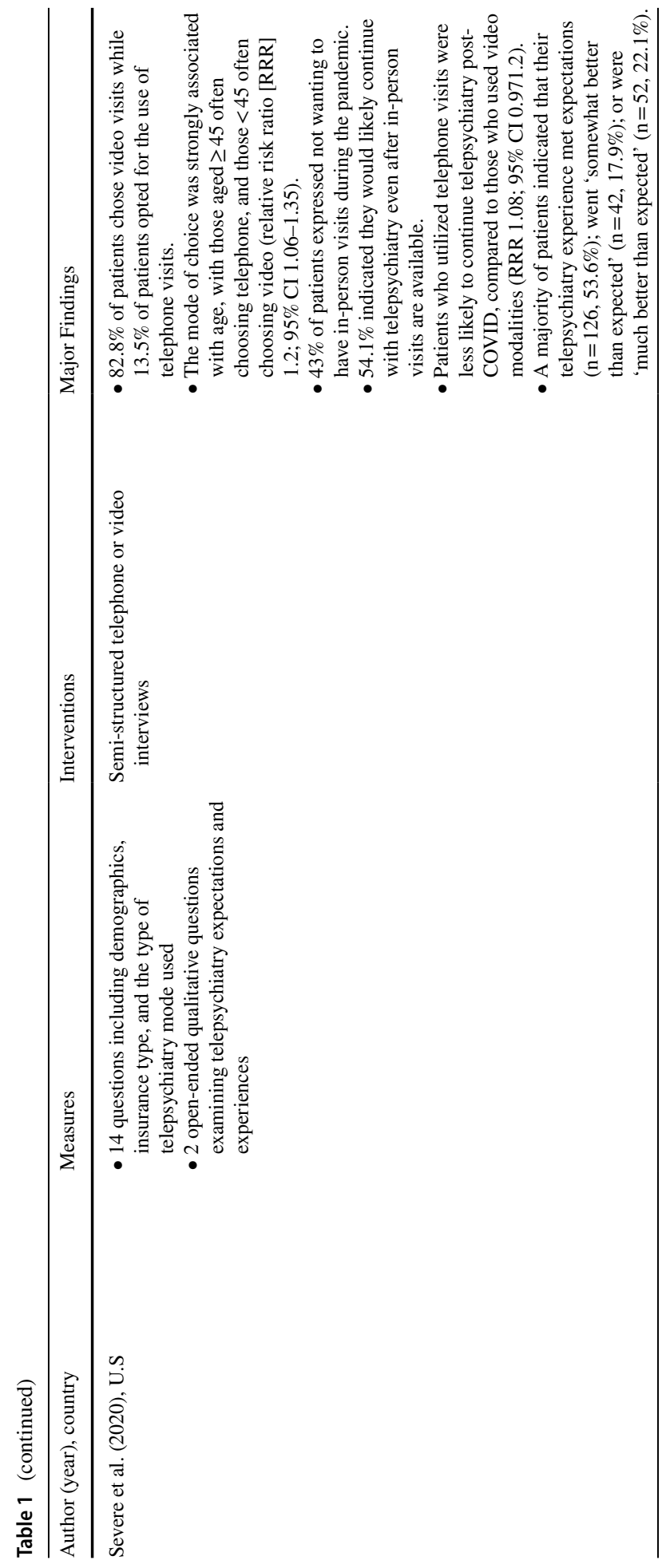




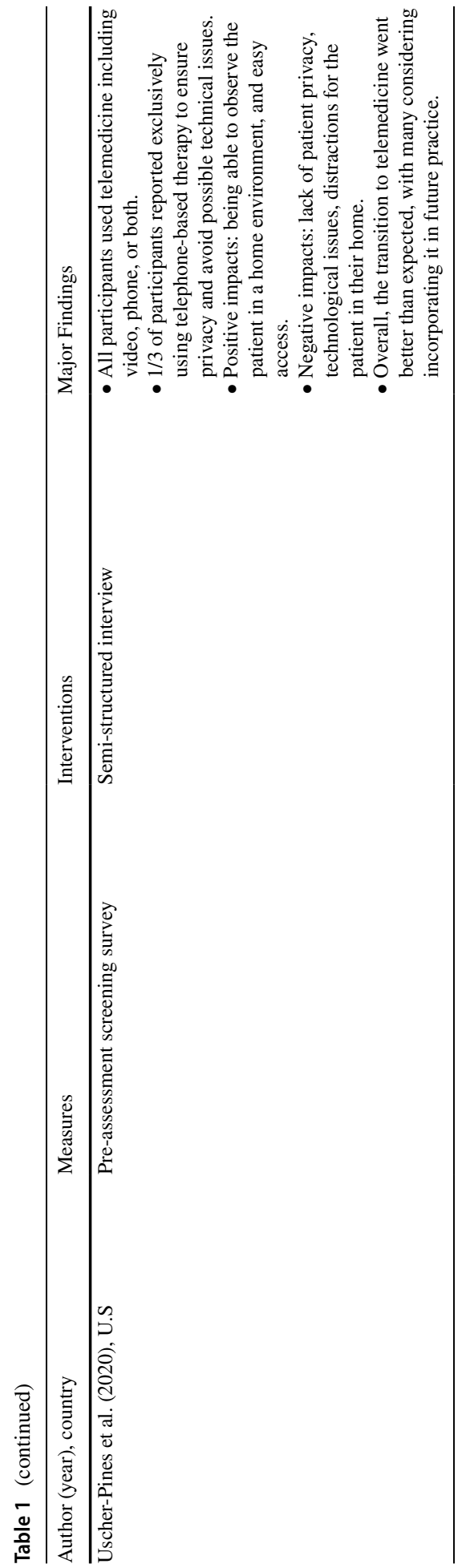

Springer 


\section{Mental Health Service Users}

Six reviewed studies examined the patients' perspectives of the transition to telepsychiatry including utilization of telepsychiatry, factors associated with using telepsychiatry, possible differences between different groups of patients, advantages and disadvantages of using telepsychiatry, and satisfaction with telepsychiatry.

Unitization of Telepsychiatry Mishkind et al. [10] examined the rapid conversion to telemental health (TMH) services during COVID-19 with a focus on the number of consultation appointments and rate of "no-show" before and after TMH implemented in the U.S.. Training and education on TMH were provided to patients and clinicians including test calls to help patients become familiar and comfortable with TMH, as well as tailored staff training. A total of 499 appointments were scheduled in the two weeks before starting TMH (March 2-13, 2020) including 84 TMH appointments, of which 442 (88.6\%) were completed and 57 (11.4\%) were no-show including $14 \mathrm{TMH}$ appointments. During the two weeks immediately following TMH implementation (March 16-27, 2020), 446 appointments were scheduled, which represents a $10.6 \%$ decreased in comparison with two weeks earlier with 32 (7.2\%) appointments being no-show. However, after the initial dip in scheduled appointments, at four weeks of TMH, appointments increased to 490 with a $7.8 \%$ noshow. From six months of April to September of 2020, the average of monthly scheduled appointments increased to $1,168(17.8 \%)$ with no-show rates decreased to a range of 5.5$8.5 \%$, leading to a $26.2 \%$ increase in completed visits. The authors suggested that telepsychiatry can be an effective way to deliver mental health services during COVID-19 and in the foreseeable future.

Variables Associated with Using Telepsychiatry A survey was conducted to examine possible factors that may influence patients' initial decision on using telepsychiatry immediately after the implementation of a stay-at-home order in the U.S. due to Covid19 [14]. A total of 212 patients and 32 patient proxies or parents, who or whose children had in-person appointments scheduled within the first three weeks of stay-at-home order, participated in the survey between June and August 2020 [14]. A majority of participants decided to use video consultation (82.8\%), while $13.5 \%$ opted for telephone, and $1.2 \%$ chose to delay their appointment until in-person visits available. Age was found to be a strong contributing factor in the mode of choice, with those aged $\geq 45$ often choosing telephone visits, and those $<45$ often choosing video modalities (relative risk ratio (RRR) $1.2 ; 95 \% \mathrm{CI}$ 1.06-1.35). Participants' gender, race, type of insurance, and number of previous visits were not significantly associated with their initial decision on adopting telepsychiatry care.

Peralta and Taveras [17] found that among 6800 telephone mental health consultations from March 25 to May 17, 2000, via a special telephone service organized by governmental and private agencies in the Dominican Republic, $67.3 \%$ of service users were female, $77.8 \%$ were adults aged $18-59$, and $27 \%$ were medical workers. The most common issues for the consultation were anxiety, sleep issues, and depression.

Patients with SMI vs. Patients Without SMI Miu et al. [11] investigated whether there were group differences between patients with severe mental illness (SMI) and non-SMI in converting face-to-face therapy to teletherapy, and between new patients with SMI and non-SMI starting therapy via telehealth during COVID-19. The study revealed that overall there was no difference between SMI and non-SMI groups in conversion rates $(\chi 2$ 
$(1)=0.471, p=0.492)$, however, older patients with SMI showed significantly decreased conversion rate $(B=-0.010, p=0.013$, OR $=0.990)$. Patients with SMI used teletherapy at much higher rates during the pandemic (SMI: mean $=1.47, S D=2.01$; non-SMI: mean $=1.04, S D=1.42 ; \mathrm{t}(251,154)=-3.027, p=0.003)$. There were no significant differences between new patients with SMI and new patients without SMI in using telehealth during the pandemic $(\chi 2(1)=1.205, p=0.272)$, and current use of teletherapy was not linked with previous engagement with teletherapy $(B=0.007, p=0.428, O R=1.007)$, indicating that experience did not play a role in utilizing teletherapy.

Advantages and Disadvantages Reviewed studies found that the implementation of telepsychiatry increased access to mental health services, especially where geographic and financial issues were a barrier [10, 17]. Peralta and Taveras [17] reported that among 6800 mental health consultations, only $5.3 \%$ of service users needed crisis interventions via face-to-face follow-up. Some patients felt that telepsychiatry was less intimidating, and the psychiatric team made good decisions on their treatment plans via virtual consultation, while others reported that interaction with the psychiatric team was impersonal, and felt they were being rushed [12].

Satisfaction with Telepsychiatry Haxhihamza et al. [16] assessed patient satisfaction with the transition to telemedicine in the wake of COVID-19. A total of 28 patients of the daily hospital in Spain participated in the survey using the short form patient satisfaction questionnaire (PSQ-18), which assesses general satisfaction, technical quality, interpersonal manner, communication, financial aspects, time spent with doctors, and accessibility and convenience. Overall patient satisfaction with the use of telemedicine was high (80.22\%), while "financial aspects" and "accessibility and convenience" scored the highest averages. Gender, age, and place of living were not found to be significant factors for the level of satisfaction with telepsychiatry services.

In the study by Severe et al. [14], 54.1\% of patients indicated they would likely continue with telepsychiatry even after in-person visits were made available, while patients who utilized telephone consultation exclusively were less likely to see themselves as using telepsychiatry compared to those who used video ( $R R R=1.08 ; 95 \% \mathrm{CI}$ : 0.97-1.2). A majority of patients reported that the telepsychiatry experience met their expectations $(n=126$, $53.6 \%)$, went 'somewhat better than expected' $(n=42,17.9 \%)$ or were 'much better than expected' $(n=52,22.1 \%)$ [14].

\section{Mental Health Service Providers}

Two reviewed studies investigated the transition to telepsychiatry from the perspectives of mental health providers including psychiatrists and psychologists.

In a qualitative study, Uscher-Pines et al. [15] examined the experiences of 20 outpatient psychiatrists using telemedicine including video, phone, or both in five U.S. states where had significantly higher COVID-19 cases during the early COVID-19 pandemic. The study was conducted by interviewing psychiatrists who had used telemedicine for 2-4 weeks, and found that less than half of participants had some experience with telemedicine prior to the pandemic. The emerging themes of the findings include (1) Utilization of telemedicine. Only $1 / 4$ of participants provided consultation for any patients in person, while $1 / 3$ of 
participants reported exclusively using telephone-based therapy to ensure privacy, avoid possible technical issues, and serve underserved patients who didn't have access to the internet and/or compatible devices, in addition, the psychiatrists were cognizant that some patients might be self-conscious about their appearance, and had social anxiety disorders. (2) Impacts of telemedicine on psychiatrist-patient interactions. Advantages of using telemedicine were identified as being able to observe the patient in a home environment, patients being more relaxed, and easier access to services, while disadvantages included lack of patient privacy, technological issues, distractions for the patient in their home, inability to do a physical exam, inadequate information to support diagnosis, and consultation time shortened. (3) Positive responses from patients. A majority of patients reported positively in regards to the transition to telemedicine, however, some psychiatrists suggested the positive response may be due to fears of ongoing access to emergency care or services being canceled. (4) Sustainability. Even though most psychiatrists accepted new patients and evaluated them via phone or video successfully, some participants voiced challenges in engaging with new patients via telemedicine including evaluating patients via phone. (5) Telemedicine after the pandemic. A strong preference to return to in-person consultation after COVID-19 was conveyed among psychiatrists even though the transition to telemedicine went better than expected among participants, with many considering incorporating it in the future. The cited reasons for in-person preference included the routine of going to an office where is a private and safe space, and concerns of substandard quality of physician-patient interactions via telemedicine.

In a U.S. national study, Pierce et al. [13] investigated 2,619 licensed practicing psychologists' telepsychology use before, during, and post COVID-19, and their relation to demographic, training, policy, and clinical practice variables. The study found that preCOVID-19, only $7.1 \%$ of psychologists conducted their clinical work using telepsychology, which increased to $85.5 \%$ during COVID-19, with $67.3 \%$ of them performing all of their clinical practice via telepsychology, while $35.0 \%$ of psychologists reported continuing to utilize telepsychology post-pandemic. The rate of using telepsychology was found to be associated with where psychologists work, for example, psychologists in outpatient treatment facilities and academic medical centers saw 26 times and 23 times growth rates, respectively, while there was 7 times increase among psychologists who work in Veteran Affairs medical centers. The highest increase of using telepsychology was found in female psychologists, those with telepsychology training and organizational support for telepsychology use, and those who treat patients with relationship issues, and anxiety. The lowest increase of adopting telepsychology was among psychologists who work in rural areas, treat antisocial personality disorders and patients with rehabilitation needs, and conduct testing and evaluation.

\section{Discussion}

The current study reviewed the literature on the transition of mental health service delivery to telepsychiatry from the perspectives of both patients and healthcare providers in response to COVID-19. Overall, a majority of patients and healthcare providers rated the transition as "met expectations" or "better than expected" despite some concerns and negative views. 
The findings of our review are in agreement with a review of 134 telepsychiatry literature prior to COVID-19 [18], in which most studies provided substantial evidence underlining that telepsychiatry can be a viable delivery method for mental health services, and a majority of patients and healthcare providers were satisfied with telepsychiatry. The same review found that telepsychiatry is comparable to in-person services in regards to the reliability of clinical assessments and treatment outcomes. In addition, a majority of reviewed studies suggest that telepsychiatry is more cost-effective in comparison with in-person services [18].

The pandemic has dramatically sped up the slow adoption of telehealth visits, and increased knowledge of what technology can offer. This forced uptake has clearly demonstrated the potential in virtual healthcare service delivery during COVID-19 and the future. Telepsychiatry has been shown to be feasible and appropriate for supporting patients and healthcare providers during COVID-19. Furthermore, geographical barriers to delivering mental health services have been broken in terms of physical distance and its associated financial burdens. In this review, some patients and healthcare providers expressed the desire to continue with telepsychiatry after in-person consultation available, while others preferred to go back to in-person services for varying reasons.

Even though evidence highlights the advantages of telepsychiatry, social determinants of health also play a critical role in service delivery. For example, some patients with chronic mental health illness must also manage socio-economic challenges, thus cannot afford to prioritize digital connectivity and the needed devices for ongoing telecare. Older patients may face similar budgetary concerns along with additional difficulties in using technology. For these groups, their primary means of communication is the phone, and this was reflected in this review as most patients who used phone consultations were 45 years old or older, and conveyed a strong desire of going back to in-person consultation in post-pandemic. Interactions between patients and psychiatrists/psychologists in mental status assessment and therapy partly rely on non-verbal cues, observable affects, body language, and behaviors, especially in the diagnosis of new patients, thus some psychiatrists in this review felt uncertain about the reliability of their assessment via telepsychiatry, particularly using the phone. Healthcare providers reported concerns about potential poor therapeutic rapport with patients when using telepsychiatry only [18]. To accommodate different needs, and improve the quality of services, a 'hybrid' model of combining both in-person and telepsychiatry in mental health service delivery has been suggested for postpandemic [19].

A majority of reviewed studies in this review had a focus on the transition of psychiatry treatments or consultations, with very limited contribution by psychotherapy. Psychotherapy has played an important role in the treatment of mental health illness and helping patients in their recovery journey [20]. In a study conducted prior to COVID-19 [19], the effectiveness and outcomes of using videoconferencing psychotherapy (VCP) were examined via a non-inferiority trial at post-treatment and follow-up among patients with panic disorder and agoraphobia, and findings show significant improvements in fear of sensations, panic, agoraphobia, and depressive symptoms, and large effect-sizes from pre to post-treatment, indicating that VCP is just as effective as in-person therapy.

In addition to phone and/or videoconference, more methods are likely to be increasingly used in future telepsychiatry including mobile apps, instant messaging, virtual reality, and artificial intelligence solutions. There is an urgent need to establish standard protocols and practice guidelines for the implementation of telepsychiatry including guidelines for privacy and confidentiality [5]. While facilitating patient learning to master different technologies used in telepsychiatry, continued development among healthcare providers, including 
those in training such as medical students, residents, and nurses is important especially in regards to privacy and confidentiality while highlighting both the opportunities and challenges of using telepsychiatry [21]. In this digital era, mental healthcare providers can play an essential role in the development of strategies and procedures to ensure that the technologies serve the purpose of providing healthcare services and enhancing therapeutic relationships while remaining their scientific rigor [21].

\section{Limitations}

There are several limitations in this review. First, there was a paucity of studies available (only eight reviewed articles) that limited our ability to discuss the topic from a broader perspective. Second, methodology challenges were identified among reviewed studies. Several reviewed quantitative studies only provided descriptive data, there was no longitudinal study to investigate longer-term effects of telepsychiatry, and the impact of the transition on patients' wellbeing was not addressed, which may be due to rapid research in nature. Third, there was a lack of research that examined other healthcare providers' perspectives such as social workers, mental health nurses, and pharmacologists as patients with mental health disorders require a multidisciplinary team to support them and help them manage their symptoms in the community. Finally, our database search was limited to articles that were peer-reviewed and published in English, which may limit views on the context of the transition of mental health services to telepsychiatry during COVID-19.

\section{Conclusion}

The current review found that the transition of mental health service delivery from faceto-face to telepsychiatry during COVID-19 met the expectations of most patients and healthcare providers, and telepsychiatry can be a feasible and appropriate method to support patients and healthcare providers. A hybrid model of combining both in-person and telepsychiatry services would meet various needs among patients and healthcare providers in the post-pandemic years ahead. Due to the time-limited nature of reviewed studies, future cross-sectional and longitudinal, and qualitative studies of telepsychiatry including patients' experience, needs evaluation, diagnosis reliability and therapeutic efficiency are required.

Acknowledgements The authors would like to thank Kayla Arisman for assisting in reviewing search results.

Author Contributions Hua Li contributed to the design of the review and search strategies, data screen and selection, data synthesis, and wrote the majority of the first manuscript draft. Alana Glecia contributed to search strategies, database search, data screen and selection, and wrote the methods section of the first manuscript draft. Arlene Kent-Wilkinson, Don Leidl, Manal Kleib, and Tracie Risling contributed to the revision of the first manuscript draft, interpretation of the results, and expertise on the subject. All authors commented on previous versions of the manuscript. All authors read and approved the final manuscript.

Funding This review is funded by the University of Saskatchewan Faculty Recruitment and Retention Fund (HL). 


\section{Declarations}

Conflict of Interest The authors declare that they have no conflicts of interest.

\section{References}

1. WHO. WHO Director-General's opening remarks at the media briefing on COVID-19 -11 March 2020, 2020. https://www.who.int/director-general/speeches/detail/who-director-general-s-opening-remarks-atthe-media-briefing-on-covid-19---11-march-2020.

2. Alavi Z, Haque R, Felzer-Kim IT, Lewicki T, Haque A, Mormann M. Implementing COVID-19 Mitigation in the Community Mental Health Setting: March 2020 and Lessons Learned. Community Ment Health J. 2020;57(1):1-7. https://doi.org/10.1007/s10597-020-00677-6.

3. Darkins A, Darkins AW, Cary MA, Cary M. Telemedicine and telehealth: principles, policies, performances and pitfalls, Springer publishing company. 2000.

4. Malhotra N, Sakthivel P, Gupta N, Nischal N, Ish P. Telemedicine: a new normal in COVID era; perspective from a developing nation. Postgrad Med J. 2020. https://doi.org/10.1136/postgradmedj-2020-138742.

5. Di Carlo F, Sociali A, Picutti E, Pettorruso M, Vellante F, Verrastro V, Martinotti G, di Giannantonio M. Telepsychiatry and other cutting-edge technologies in COVID-19 pandemic: Bridging the distance in mental health assistance. Int J Clint Pract. 2021;75(1). https://doi.org/10.1111/ijcp.13716.

6. Barnett ML, Ray KN, Souza J, Mehrotra A. Trends in telemedicine use in a large commercially insured population, 2005-2017. JAMA. 2018;320(20):2147-9. https://doi.org/10.1001/jama.2018.12354.

7. WHO. Draft global strategy on digital health 2020-2025, 2019. https://www.who.int/docs/defaultsource/documents/gs4dhdaa2a9f352b0445bafbc79ca799dce4d.pdf.

8. Wynne B, LaRosa J. A Tell-All on Telehealth: Where Is Congress Heading Next?, 2019. https://www. commonwealthfund.org/blog/2019/telehealth-where-congress-heading-next.

9. CHI. Connecting patients for better health: 2018, 2016. https://www.infoway-inforoute.ca/en/component/ edocman/resources/3152-connecting-patients-for-better-health-2016.

10. Mishkind M, Shore JH, Bishop K, D’Amato K, Brame A, Thomas M, Schneck CD. Rapid conversion to telemental health services in response to COVID-19: experiences of two outpatient mental health clinics. Telemedicine and e-Health. 2020. https://doi.org/10.1089/tmj.2020.0304.

11. Miu AS, Vo HT, Palka JM, Glowacki CR, Robinson RJ. Teletherapy with serious mental illness populations during COVID-19: telehealth conversion and engagement, Counselling Psychology Quarterly 2020;1-18. https://doi.org/10.1080/09515070.2020.1791800.

12. Heyman-Kantor R, Hardy N, Corcoran AR. Patient Perspectives on Telepsychiatry on the Inpatient Psychiatric Unit During the COVID-19 Pandemic. Journal of Patient Experience. 2020;7(5):677-9. https://doi.org/10.1177/2374373520958519.

13. Pierce BS, Perrin PB, Tyler CM, McKee GB, Watson JD. The COVID-19 telepsychology revolution: A national study of pandemic-based changes in US mental health care delivery. Am Psychol. 2020.

14. Severe J, Tang R, Horbatch F, Onishchenko R, Naini V, Blazek MC. Factors Influencing Patients' Initial Decisions Regarding Telepsychiatry Participation During the COVID-19 Pandemic: TelephoneBased Survey. JMIR formative research. 2020;4(12):e25469.

15. Uscher-Pines L, Sousa J, Raja P, Mehrotra A, Barnett ML, Huskamp HA. Suddenly becoming a "virtual doctor": Experiences of psychiatrists transitioning to telemedicine during the COVID-19 pandemic. Psychiatr Serv. 2020;71(11):1143-50. https://doi.org/10.1176/appi.ps.202000250.

16. Haxhihamza K, Arsova S, Bajraktarov S, Kalpak G, Stefanovski B, Novotni A, Milutinovic M. Patient satisfaction with use of telemedicine in university clinic of psychiatry: Skopje, North Macedonia during COVID-19 pandemic. Telemedicine and e-Health. 2020. https://doi.org/10.1089/tmj.2020.0256.

17. Peralta EA, Taveras M. Effectiveness of teleconsultation use in access to mental health services during the coronavirus disease 2019 pandemic in the Dominican Republic. Indian J Psychiatry. 2020;62(Suppl 3):S492. https://doi.org/10.4103/psychiatry.IndianJPsychiatry_1047_20.

18. Hubley S, Lynch SB, Schneck C, Thomas M, Shore J. Review of key telepsychiatry outcomes, World journal of psychiatry 2016;6(2):269. https://doi.org/10.5498/wjp.v6.i2.269.

19. Bouchard S, Allard M, Robillard G, Dumoulin S, Guitard T, Loranger C, Green-Demers I, Marchand A, Renaud P, Cournoyer LG. Videoconferencing psychotherapy for panic disorder and 
agoraphobia: Outcome and treatment processes from a non-randomized non-inferiority trial. Front Psychol. 2020;11:2164. https://doi.org/10.3389/fpsyg.2020.02164.

20. Dereboy Ç, Şenel H, Öztürk CŞ, Şakiroğlu M, Eskin M. Psychotherapy: Playing the Three Monkeys in Mental Health Service Provision?, Archives of Neuropsychiatry 2017;54(4):301. https://doi.org/10. 5152/npa.2017.13785.

21. Smith K, Ostinelli E, Macdonald O, Cipriani A. Covid-19 and telepsychiatry: development of evidence-based guidance for clinicians. JMIR Mental Health. 2020;7(8):e21108. https://doi.org/10.2196/ 21108.

Publisher's Note Springer Nature remains neutral with regard to jurisdictional claims in published maps and institutional affiliations. 